\title{
Einstein et la marche du monde - idéaliste engagé, parfois prémonitoire
}

Jean Martin

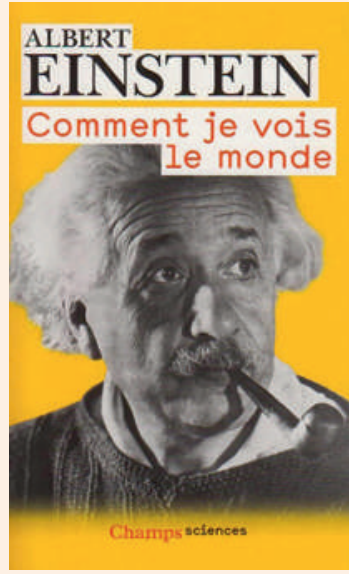

Albert Einstein

Comment je vois le monde

Paris: Flammarion; 2009

(dernière édition).

247 pages. $7.20 €$.

ISBN 978-2-0812-2904-4

jean.martin[at]saez.ch
Lecture de vacances... Je me suis plongé dans un recueil d'Albert Einstein (1879-1955), grand homme de la physique théorique, découvreur des lois de la relativité, prix Nobel 1921 - et qui a eu la nationalité suisse de 1901 à sa mort. Il s'agit de textes écrits entre 1930 et 1935 qui méritent d'être lus. Cinq chapitres: le premier intitulé «Comment je vois le monde», les suivants traitant de son pacifisme affirmé, de son engagement contre le national-socialisme (alors que beaucoup restaient timorés), de son attachement à la communauté juive. Le dernier est constitué d'articles et exposés sur des sujets scientifiques.

Illustrations substantielles de ce qu'Einstein se sentait citoyen du monde et était très soucieux de l'instauration d'une convivialité planétaire, en étant notamment un adversaire de toute guerre. Véritable «honnête homme». Avec des notations et avertissements qui restent très actuels.

\section{Vivre - Pratique, éthique, rapport à soi}

«Je n'ai jamais considéré le plaisir et le bonheur comme une fin en soi. En revanche des idéaux ont suscité mes efforts et m'ont permis de vivre. Ils s'appellent le bien, le beau, le vrai [...] Si je ne me m'obstine pas inlassablement à poursuivre cet idéal, pourtant inaccessible, la vie n'a aucun sens pour moi. Or, l'humanité se passionne pour des buts dérisoires, la richesse, la gloire, le luxe.»

«Chaque jour, mille fois, je ressens ma vie, corps et âme, intégralement tributaire du travail des vivants et des morts. Je voudrais donner autant que je reçois et je ne cesse de recevoir.» «La joie de contempler et de comprendre, voilà le langage que me porte la nature.»

«La vertu républicaine correspond à mon idéal politique. Chaque vie incarne la dignité de la personne humaine, et aucun destin ne justifierait une quelconque exaltation de quiconque. Or, le hasard s'amuse de moi, car les hommes me témoignent une invraisemblable et excessive admiration.»

Question de la désobéissance civile. «Je me pose une très vieille question. Que dois-je faire quand l'Etat exige de moi un acte inadmissible, une attitude que ma conscience rejette? Je suis totalement dépendant de la société où je vis, donc je devrais me soumettre?... Cette pensée dément violemment le sentiment inné de la justice [...] Je dois réveiller et soutenir ce sentiment de responsabilité morale.»

Einstein parle de religion, évoque un concept de religiosité cosmique: «Les génies religieux se sont distingués par cette religiosité face au cosmos. Elle ne connaît ni dogme ni Dieu conçu à l'image de l'homme. Nous imaginons que les hérétiques de tous les temps se nourrissaient de cette forme de religion; ils étaient souvent suspectés d'athéisme, mais parfois aussi de sainteté. Considérés ainsi, Démocrite, François d'Assise, Spinoza, se ressemblent profondément.»

\section{Le pacifiste - A propos de nationalisme, de politique, de droits des gens}

«Je hais violemment l'héroïsme sur commande, la violence gratuite et le nationalisme débile. La guerre est la chose la plus méprisable.»

«N'importe où, en quinze jours, une campagne de presse peut exciter une population incapable de jugement à un tel degré de folie que les hommes sont prêts à s'habiller en soldats pour tuer et se faire tuer.» Quatre-vingt ans plus tard, les effets potentiels des campagnes médiatiques d'un type ou de l'autre restent comparables, mutatis mutandis. A quoi s'ajoutent internet et la galaxie des réseaux sociaux, qui peuvent exciter une population dans le monde entier, et en quinze heures plutôt que quinze jours.

«Les politiciens réactionnaires ont réussi, en agitant le spectre d'un danger extérieur, à sensibiliser l'opinion publique contre toutes les activités des intellectuels. Ils essaient maintenant d'interdire la liberté de l'enseignement. Que doit faire la minorité intellectuelle contre ce mal? Je ne vois qu'une voie possible: celle de la désobéissance et du refus de collaborer, celle de Gandhi.»

Des aphorismes. «L'effort d'unir sagesse et pouvoir aboutit rarement et seulement très brièvement.» «La majorité des imbéciles reste invincible et satisfaite en toute circonstance.» «Pour être un membre irréprochable parmi une communauté de moutons, il faut être soi-même un mouton.»

\section{Croissance économique et technologique - Des correctifs à apporter?}

«Le problème de la répartition du travail et des produits fabriqués s'avère de plus en plus difficile. Le libre jeu des forces économiques, l'effort désordonné et sans frein des individus pour acquérir et dominer, ne conduisent plus à une solution supportable (...) il s'agit d'éviter la disparition menaçante des ressources productives, l'appauvrissement et le retour à l'état sauvage d'une grande partie de la population.»

A noter que Einstein juge souhaitable un ordre planifié de la production des biens et de l'emploi de la main d'œuvre... Les années qui ont suivi ont montré les limites de ces volontés de planification (surtout en Europe orientale mais pas seulement); il 
convient ici de se souvenir de l'attrait qu'exerçait, chez beaucoup d'intellectuels occidentaux dans les années 1930-40, ce que tentaient les régimes socialistes. Le pacifisme des milieux de gauche attirait sans doute aussi la sympathie d'Einstein.

\section{Pour une gouvernance plus large que celle des} Etats-nations. Une propédeutique des esprits

De manière répétée, Einstein veut réfléchir au-delà des nationalismes belliqueux qu'il observe, pour aller vers plus de coopération et de mise en commun. «Notre continent ne peut surmonter ses problèmes qu'en dépassant ses traditionnels conflits de systèmes politiques. L'organisation européenne se renforcerait et la suppression des barrières douanières s'intensifierait. Cet objectif supérieur ne dépend pas de simples conventions étatiques. Il y faut d'abord une propédeutique des esprits. Eveillons chez les hommes un sentiment de solidarité qui ne s'arrête pas aux frontières.» «Chaque peuple doit comprendre et vouloir les limitations nécessaires de son droit de souveraineté (...) Aura-t-on besoin encore de nouvelles catastrophes pour inciter les Etats à s'engager [dans ce sens]?» Disant cela, Einstein veut principalement éloigner le spectre de la guerre; aujourd'hui, ce besoin de transfert de parties de la souveraineté des Etats, incluant le respect des droits et intérêts des autres, est très actuel au plan de la survie «durable» de la société humaine et de la planète.

Il appelle de ses vœux la mise sur pied de Commissions internationales de justice et d'arbitrage, dont les Etats s'engageraient formellement et irrévocablement à suivre les arrêts. Idéaliste/irréaliste, ce qui s'est passé depuis lors n'autorise guère à y croire - même si la Cour pénale internationale a été créée et a montré récemment une certaine efficacité.

\section{Une Terre désormais trop petite - Préoccupa- tions d'équilibre écologique et humain}

«Si la bombe H est réalisée, elle entraînera la contamination radioactive de l'atmosphère et l'anéantissement de toute vie sur la terre aussi loin que la technique le rendra possible. L'horreur dans cette escalade consiste en son apparente inéluctabilité.» Peut-on dire, un demi-siècle plus tard, que les efforts en partie réalisés de limitation de l'armement atomique sont une raison d'espérer?*

Quand nous aurons triomphé de cette obsession [psychose sécuritaire, alimentée par ce qu'on appelle aujourd'hui le complexe militaro-industriel], nous pourrons aborder le véritable problème politique: Comment assurer sur une terre désormais trop petite l'existence et les relations humaines?»

Souci que chacun puisse avoir un emploi et le sentiment d'être utile - et que les personnes âgées bénéficient d'une sécurité: «Il faut absolument veiller à ce que les jeunes puissent prendre part aux processus de production. Les vieux doivent être exclus (sic!) de certains travaux et recevoir en compensation une certaine rente puisque, jadis, ils ont fourni un travail productif reconnu par la société.»

Le racisme est aussi pour lui une grande préoccupation. Après s'être installé aux Etats-Unis, il s'est fortement engagé pour la cause des Noirs américains et les droits civiques: «Les minorités, en particulier celles dont les traits physiques sont reconnaissables, sont considérées par les majorités comme des classes inférieures de l'humanité. Les victimes d'une telle horreur s'imprègnent à leur tour elles-mêmes de ce préjugé de race et elles se mettent à considérer d'autres semblables comme des inférieurs.»

\section{Proche-Orient}

Einstein a été un grand soutien de l'implantation juive en Palestine. Il a contribué à la fondation de l'Université hébraïque de Jérusalem. On lui a même proposé en 1952 de devenir Président de l'Etat d'Israël. Sa conscience d'appartenir à la communauté juive (qu'il voyait comme une communauté culturelle et intellectuelle plutôt que politique, il y insiste plusieurs fois) s'est renforcée tout au long de sa vie.

Il écrit vers 1932: «Les forces du peuple juif ont réalisé en Palestine une œuvre de construction magnifique. Nous avons surmonté la dure épreuve que les évènements des dernières années nous ont imposée (...) Mais n'oublions jamais la leçon de cette crise. L'établissement d'une coopération satisfaisante entre Juifs et Arabes n'est pas le problème de l'Angleterre mais le nôtre. Nous devons nous entendre nousmêmes sur les lignes directrices d'une politique de communauté efficace et adaptée aux besoins de nos deux peuples.»

Plus loin: «Pour y parvenir, nous devons résoudre, publiquement et dignement, la cohabitation avec le peuple frère des Arabes. Nous avons l'occasion de prouver ce que nous avons appris durant les siècles d'un passé durement vécu.» Intéressant - les choses ne se sont guère passées dans les décennies qui ont suivi comme l'envisageait Albert Einstein.

\section{Education et formation}

«La compréhension d'autrui ne progressera qu'avec le partage des joies et des souffrances (...) L'effrayant dilemme de la situation politique s'explique par ce péché d'omission de notre civilisation. Sans culture morale, aucune chance pour les hommes.»

«La perte de la vue d'ensemble est un immense sacrifice».«Il ne suffit pas d'apprendre à l'homme une spécialité, car il devient ainsi une machine utilisable mais non une personnalité. Il importe qu'il acquière un sentiment de ce qui vaut la peine d'être entrepris. Quand je conseille ardemment «Les Humanités», c'est cette culture vivante que je recommande. Les excès du système de compétition et de spécialisation prématurée sous le fallacieux prétexte d'efficacité assassinent l'esprit.» Einstein appelait déjà, comme beaucoup aujourd'hui, à une revalorisation des humanités et, généralement, des dimensions humanistes. 